\title{
Sliced Wigner fourth order moment spectra smoothed by $\gamma$-method and its application to detection of valve system faults in an engine
}

\author{
Sang-Kwon Lee \\ Department of Mechanical Engineering, Inha \\ University, Yong-Hyun Dong 253, Inchon, 402-751, \\ South Korea \\ Tel.: +8232860 7305; Fax: +8232868 1716; \\ E-mail: sangkwon@inha.ac.kr
}

Received 25 August 2000

\begin{abstract}
The research proposes a method for detecting valve system faults in an engine. The methodology is based on the detection of impulsive sound embedded in the high level of background noise by using the sliced Wigner fourth order moment spectra (SWFOMS) which is inherently more robust in the presence of random (Gaussian) noise. However, in previous work, the SWFOMS for multiple signals had problems with its application which were due to the existence of nonoscillating cross-terms not smoothed by conventional methods. In this paper, the $\gamma$-method is developed to smooth nonoscillation cross-terms. Finally, the techniques developed are applied to the diagnosis of valve system faults in an engine.
\end{abstract}

\section{Introduction}

In general, the impulsive noise and vibration signal generated from an engine are due to abnormal conditions of the moving components of an engine, such as the valve train mechanism, piston slap, injector and crankshaft train $[2,11,15,16,18]$. Thus, the detection of the impulsive signal is useful for extracting information about the condition monitoring of these components in an engine [14,16]. The signals measured from an engine in a normal condition are commonly complicated signals containing both narrowband and broadband components. The presence of an abnormal condition is often indicated by the presence, or increase in, impulsive signal elements [11]. These impulsive signals may be due to a change of stiffness, mass in the system or the input force of system [12], and by characterizing them one can gain insight into the likely causes of the fault. The detection of these impulsive signals is hampered by the presence of signals associated with the normal running of an engine, with the consequence that the detection of the weak impulsive signals, which are especially associated with incipient faults, is difficult. It is the 'normal' signals that form the background noise environment against which the detection of fault-induced impulsive signals must be conducted. To aid fault detection, it is valuable to enhance the impulsive signals by suppressing this background noise prior to further processing. Such pre-processing can be based upon one of several signal-processing paradigms. After successful pre-processing, the signal has an increased Signal to Noise Ratio (SNR), which makes it more amenable to one of a range of signal processing tools which can be used to characterize the signal, including Auto-Regressive (AR) modelling [10], kurtosis evaluation [3], cepstrum analysis [11], timefrequency analysis [5,20], higher order spectra [4], the two stage Adaptive Line Enhancer [16] and the sliced Wigner fourth order moment spectra (SWFOMS) [14]. The SWFOMS, in particular, has been inherently more robust in detecting impulsive signals embedded in the presence of random (Gaussian) noise.

However, the SWFOMS suffers from non-oscillating cross-terms for multicomponent signals. These crossterms cannot be smoothed by a conventional kernel function such as an exponential kernel function, which has been used for smoothing of cross-terms in the bilinear time-frequency method [8]. In this paper, in order to smooth the non-oscillating cross-terms, the SWFOMS smoothed by the $\gamma$-method has been developed and applied to the diagnosis of valve system faults in an automotive engine.

\section{Review of the sliced Wigner fourth-order moment spectra (SWFOMS)}

The general Wigner higher order moment spectra (WHOMS) of order $n+1$ for signal $s(t)$ is defined by 


$$
\begin{array}{r}
W_{4}\left(t, f_{1}, f_{2}, f_{3}\right)_{p, q, r, s}= \\
W_{s s s s}\left(t-t_{m}, f_{1}+f_{d_{3}} / 4-f_{q}, f_{2}-f_{d_{3}} / 4+f_{r}, f_{3}+f_{d_{3}} / 4-f_{s}\right) \\
\\
\times e^{j 2 \pi\left(f_{d_{3}} t+f_{1} \delta_{1}+f_{2} \delta_{2}+f_{3} \delta_{3}+f_{q}\left(\delta_{m}-\delta_{1}\right)-f_{r}\left(\delta_{m}-\delta_{2}\right)+f_{s}\left(\delta_{m}-\delta_{3}\right)-f_{p} \delta_{m}\right)} \\
p, q, r, s \in\{a, b\}
\end{array}
$$

Fonollosa [7]

$$
\begin{aligned}
& W_{n+1}\left(t, f_{1}, \ldots, f_{n}\right) \\
= & \int_{x i} S^{*}\left(\sum_{i=1}^{n} f_{i}+\frac{1}{n+1} \xi\right) . \\
& \prod_{i=1}^{n} S^{(*)^{i+1}}\left(f_{i}-\frac{1}{n+1} \xi\right) e^{-j 2 \pi \xi t} d \xi
\end{aligned}
$$

where $n=1, n=2$ and $n=3$ are the Wigner-Ville distribution (WVD), the Wigner third order moment spectrum (WTOMS) and the Wigner fourth order moment spectrum (WFOMS), respectively.

Although the WVD has significant resolution advantages over other time-frequency methods, its application is obstructed by problems associated with cross/interference terms. In the following, we present expressions for the cross-terms of the WVD and the WFOMS. Consider a signal $x(t)$ which consists of two components $s_{a}(t)$ and $s_{b}(t)$,

$$
x(t)=s_{a}(t)+s_{b}(t)
$$

Moreover, consider a specific form of the two components,

$$
\begin{aligned}
& s_{a}(t)=s\left(t-t_{a}\right) e^{j 2 \pi f_{a} t} \\
& s_{b}(t)=s\left(t-t_{b}\right) e^{j 2 \pi f_{b} t}
\end{aligned}
$$

where $s(t)$ is a prototype signal, $t_{a}$ and $t_{b}$ are time shifts and $f_{a}$ and $f_{b}$ are frequency shifts. In general, the $n+1$ th order Wigner distribution for two component signals is the sum of $2^{n+1}$ distributions, of which two are auto-terms and $2^{n+1}-2$ are cross-terms. A general expression for cross-terms in the signal of the form in Eq. (2) can be developed. These expressions are derived by simply substituting Eqs (2) and (3) into the definitions of the distributions and performing simple, but tedious, manipulations [17].

\subsection{The Wigner-ville distribution (WVD)}

$$
\begin{aligned}
& W_{2}(t, f)_{p, q} \\
= & W_{s s}\left(t-t_{m}, f-f_{m}\right) e^{j 2 \pi\left(f_{d_{1}} t+f \delta-f_{m} \delta\right)}, \\
& p, q \in\{a, b\}
\end{aligned}
$$

where $t_{m}=\left(t_{p}+t_{q}\right) / 2$, similarly $f_{m}=\left(f_{p}+\right.$ $\left.f_{q}\right) / 2, \delta=t_{q}-t_{p}, f_{d_{1}}=f_{q}-f_{p}$ and $W_{s s}(t, f)$ is the WVD of $s(t)$. By way of an example of the above notation, $W_{2}(t, f)_{a, b}(p=a, q=b)$ represents the cross-term between $s_{a}(t)$ and $s_{b}(t)$. The complete WVD of $x(t)$ is the sum of all components $W_{2}(t, f)_{p, q}$. From Eq. (4) it is clear that $W_{2}(t, f)_{a, b}=W_{2}(t, f)_{b, a}^{*}$ ensuring that the final distribution is real. Further, note that $W_{2}(t, f)_{a, a}$ and $W_{2}(t, f)_{b, b}$ are simply $W_{s s}(t, f)$ repositioned in the time-frequency plane.

It is also apparent that the cross-terms are oscillatory and that the rate of oscillation in the frequency direction ( $t=$ constant) is $\delta$ and the rate of oscillation in the time direction ( $f=$ constant) is $f_{d_{1}}$. Thus the crossterms oscillate more rapidly the further apart the signal components are. Assuming smoothing is used to reduce the cross-terms, it is then the rapid oscillations which are most easily attenuated, with the result that the crossterms between closely spaced components are more troublesome to attenuate than the cross-terms between distant components.

\subsection{The Wigner fourth order moment spectrum (WFOMS)}

where $f_{d_{3}}=\left(f_{q}+f_{s}-f_{p}-f_{r}\right), \delta_{3}=t_{p}-t_{s}, \delta_{m}=$ $\left(\delta_{1}+\delta_{2}+\delta_{3}\right) / 4$ and $W_{s s s s}\left(t, f_{1}, f_{2}, f_{3}\right)$ is the WFOMS of $s(t)$. In this case the auto-terms are non-oscillating and are located at the 'natural' position. To see this, take $p=q=r=s=a$, then $f_{d_{3}}=0$ and the auto-terms are centered on the frequency triplet $\left(f_{1}=\right.$ $f_{a}, f_{2}=f_{a}, f_{3}=f_{a}$ ).

\subsection{The sliced Wigner fourth order moment spectra}

The general Wigner higher moment spectra (WHOMS) are functions of $n+1$ variables. This presents problems when attempting to display results, since the resulting plot exists in $n+2$ dimensional space. The computation of the WHOMS also imposes a heavy computational burden, as one is required to perform $n$ dimensional FFTs for each time segment. For these reasons it is common to consider a subset of the WHOMS called the principal slice. The principal slice is defined as the only plane in which a single complex exponential appears as a Dirac delta. This plane for the WFOMS is obtained by setting $f_{1}=-f_{2}=f_{3}=f$ 
and $n=3$ in Eq. (1). It is called the SWFOMS (sliced Wigner fourth order moment spectra). This slice generally includes both auto-terms and cross-terms and as we show below, the number of cross-terms is significantly reduced.

By taking the principal slice of the WFOMS the only terms in Eq. (5) which are guaranteed to remain are those for which $r=p$ and $s=q$, so the original 14 cross-terms in the WFOMS reduce to only two in its principal slice. The principal slice version of Eq. (5) is

$$
\begin{aligned}
& W_{4}(t, f)_{p, q} \\
= & W_{s s s s}\left(t-t_{m}, f-\left(f_{p}+f_{q}\right) / 2\right) \\
= & e^{j 2 \pi\left(2 f_{d_{3}} t+2 f \delta-2 f_{m} \delta\right)}, p, q \in\{a, b\}
\end{aligned}
$$

This should be compared with the corresponding expression for the standard Wigner distribution Eq. (4). In both cases the cross-terms are located mid-way between the auto-terms and oscillate at rates proportional to the separation of signal components.

While Eq. (6) describes all the terms which necessarily lie on the principal slice, if the two frequency shifts are equal, i.e. $f_{a}=f_{b}$, then $f_{d_{3}}=0$ in which all the elements of Eq. (6) lie on the principal slice and we write

$$
\begin{aligned}
W_{4}(t, f)_{p, q, r, s}= & W_{s s s s}\left(t-t_{m}, f-f_{p}\right) \\
& e^{j 2 \pi\left(t_{p}-t_{q}+t_{r}-t_{s}\right)\left(f-f_{p}\right)}
\end{aligned}
$$

These components are located in time at quarter intervals between $t_{a}$ and $t_{b}$. None of the terms in Eq. (7) oscillates as a function of time; furthermore, for terms satisfying $t_{p}-t_{q}+t_{r}-t_{s}=0$, Eq. (7) is completely non-oscillatory. If the conventional smoothing is applied to reduce these non-oscillation cross-terms, it is will fail to attenuate non-oscillatory terms since these non-oscillatory terms are difficult to distinguish from the auto-terms [14]. Hence, the conventional smoothing is processed by multiplying the ambiguity function of the SWFOMS by kernel functions [17].

\section{Smoothing of non-oscillating cross-terms in the SWFOMS}

In order to smooth effectively the non-oscillating cross-terms in the SWFOMS, consider the frequency version of the WVD,

$$
\begin{aligned}
W(t, f)= & \int_{\xi} S^{*}\left(f+\frac{1}{2} \xi\right) S\left(f-\frac{1}{2} \xi\right) \\
& e^{-j 2 \pi \xi t} d \xi
\end{aligned}
$$

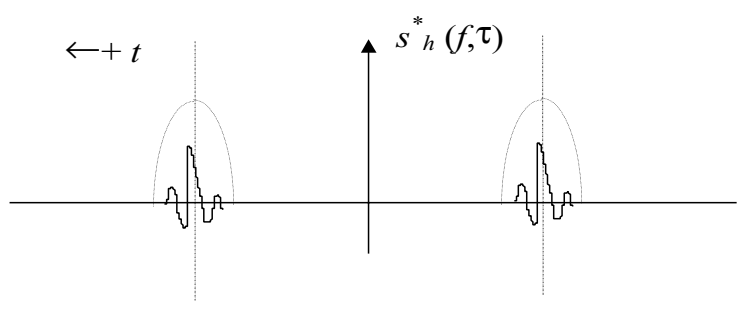

(a)

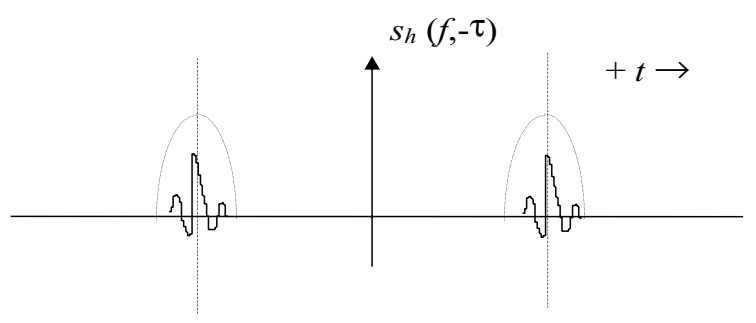

(b)

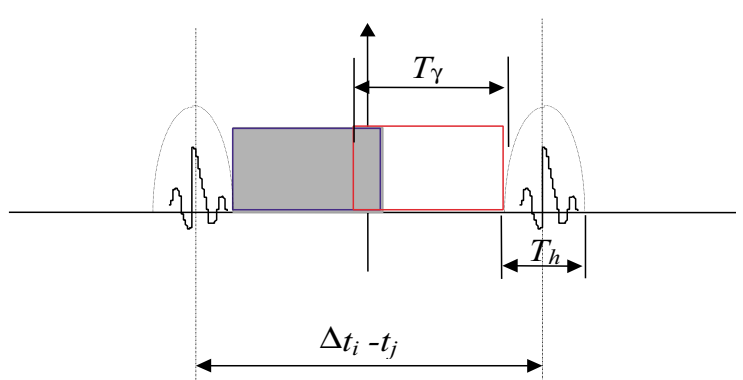

(c)

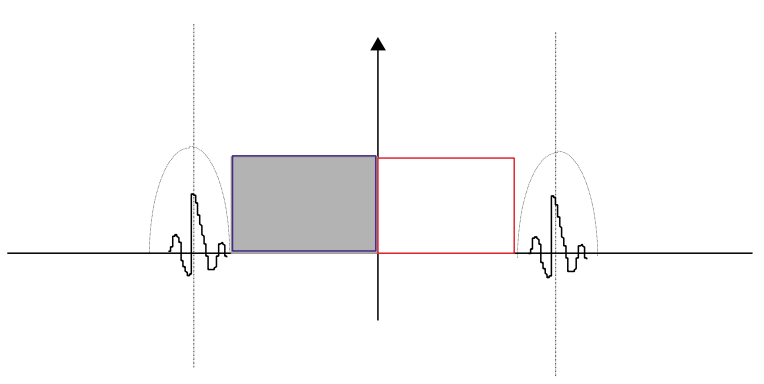

(d)

Fig. 1. Illustrating the proper width of the $\gamma(\tau)$ window function. (a) convolving signal $s_{h}^{*}(t)$. (b) convolving of signal $s_{h}(t)$ (c) $T_{\gamma}>t_{i}-t_{j} / 2-T_{h}$ (d) $T_{\gamma}<t_{i}-t_{j} / 2-T_{h}$.

The pseudo Wigner distribution (PWD) [1] can be written by using the frequency domain windowing function $H(\xi / 2)$ as follows:

$$
W_{p w}(t, f)
$$




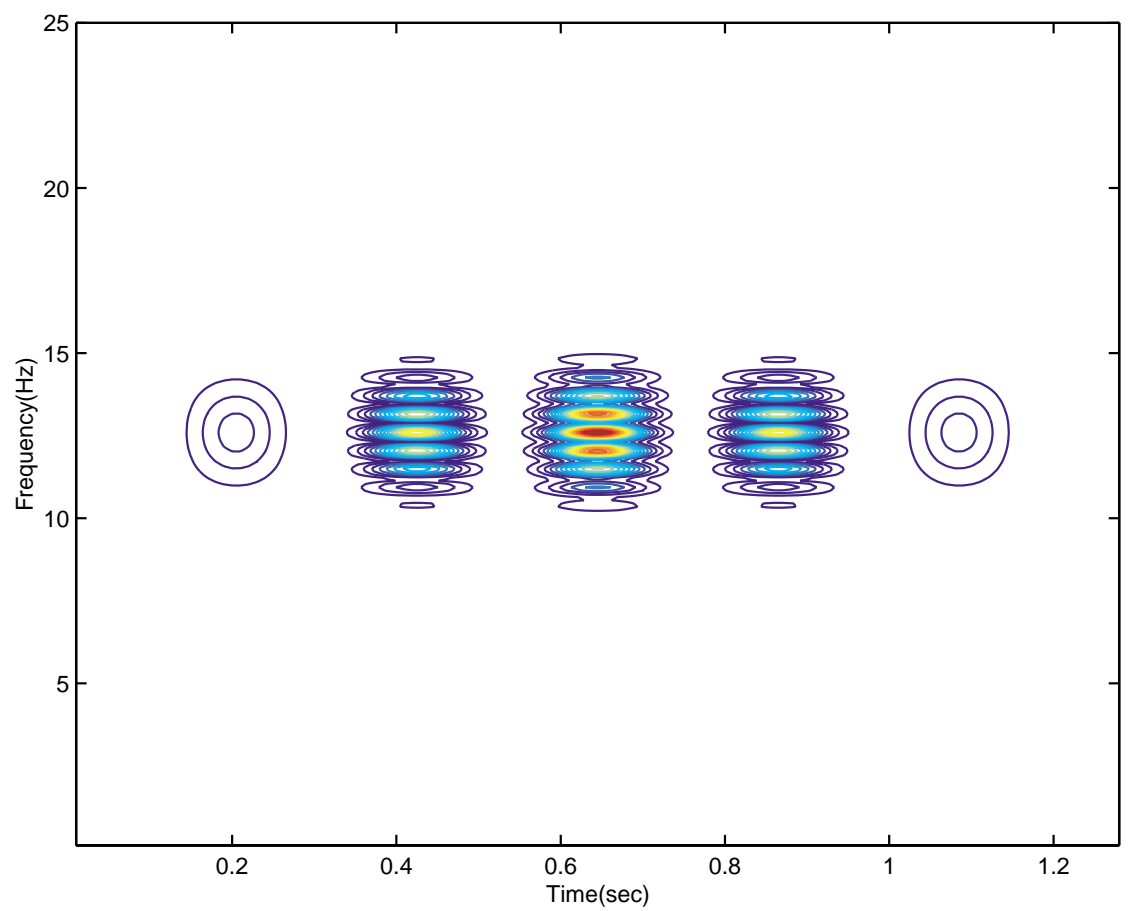

(a)

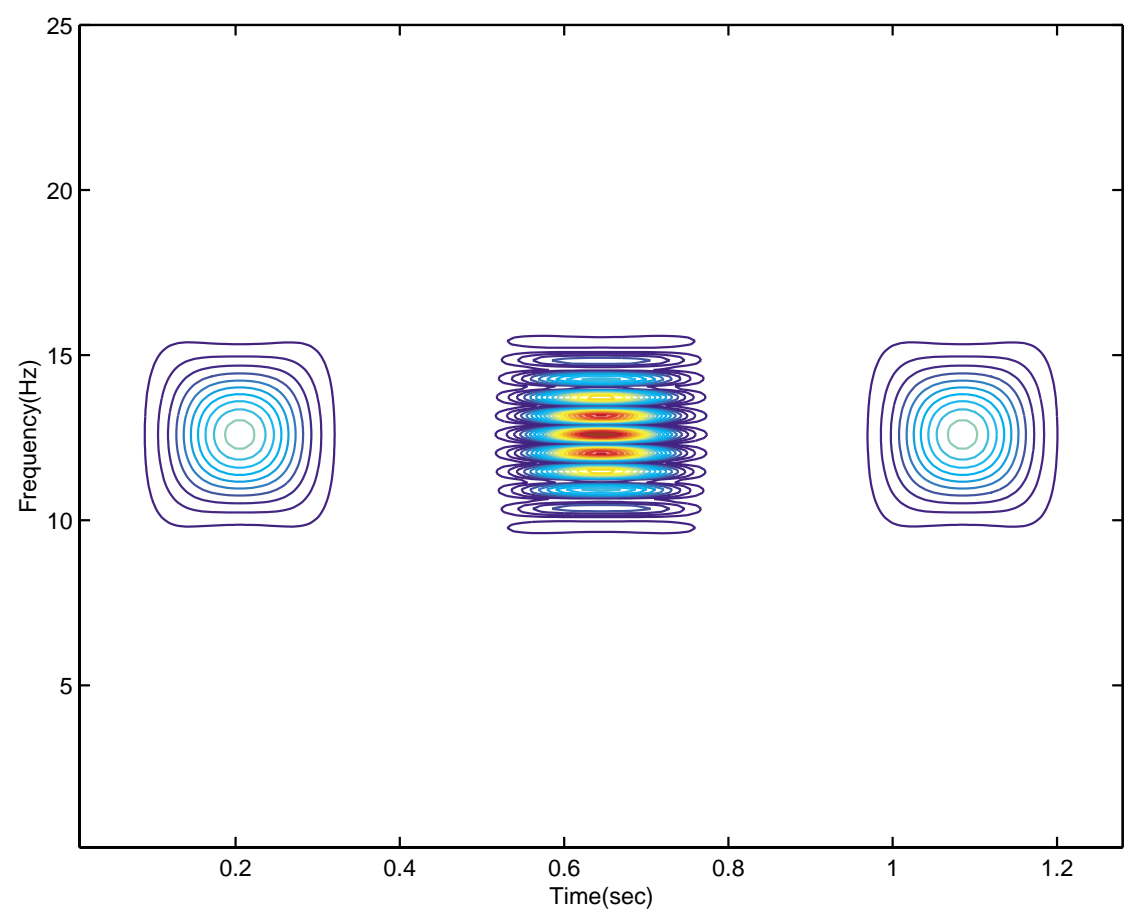

(b)

Fig. 2. Comparison between the WVD and SWFOMS for the two component signals centered at $0.2 \mathrm{~s}$ and $1.08 \mathrm{~s}$ with $12.5 \mathrm{~Hz}$. (a) SWFOMS. (b) WVD. 


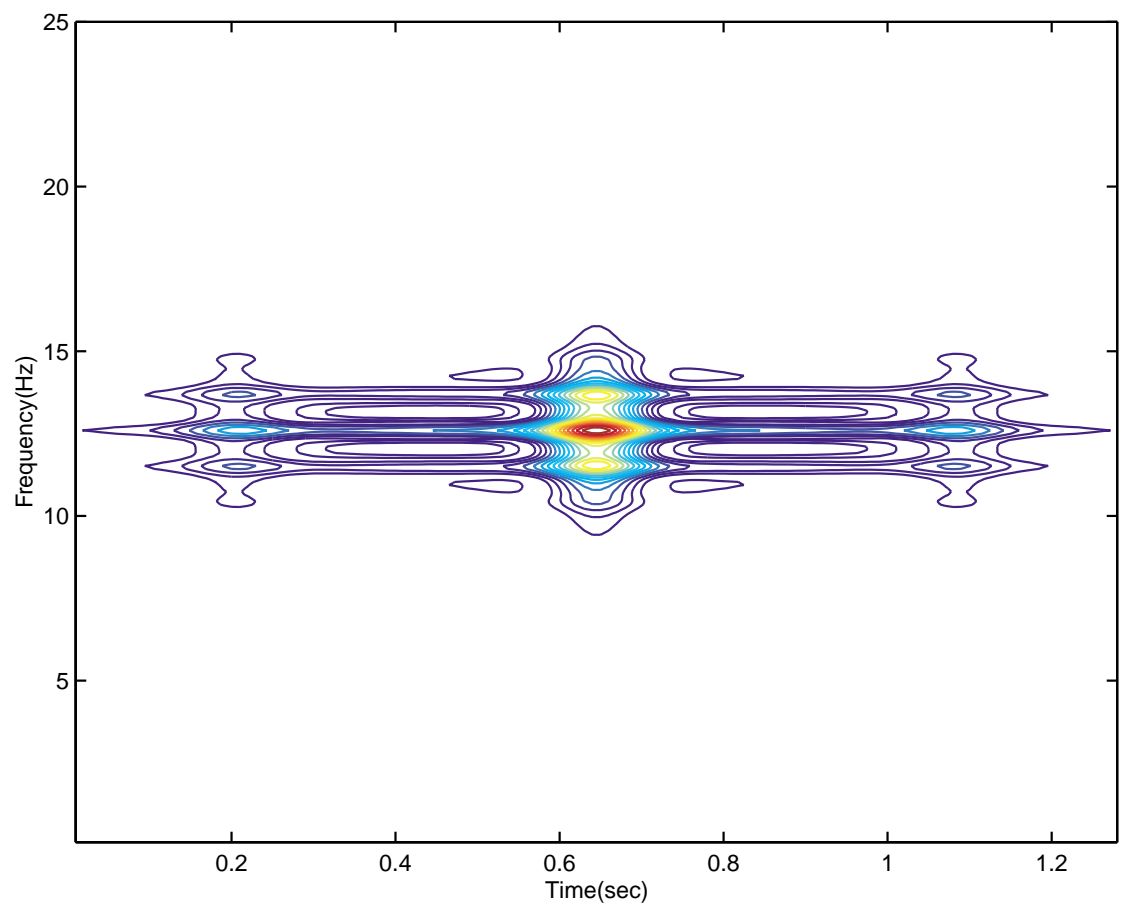

(a)

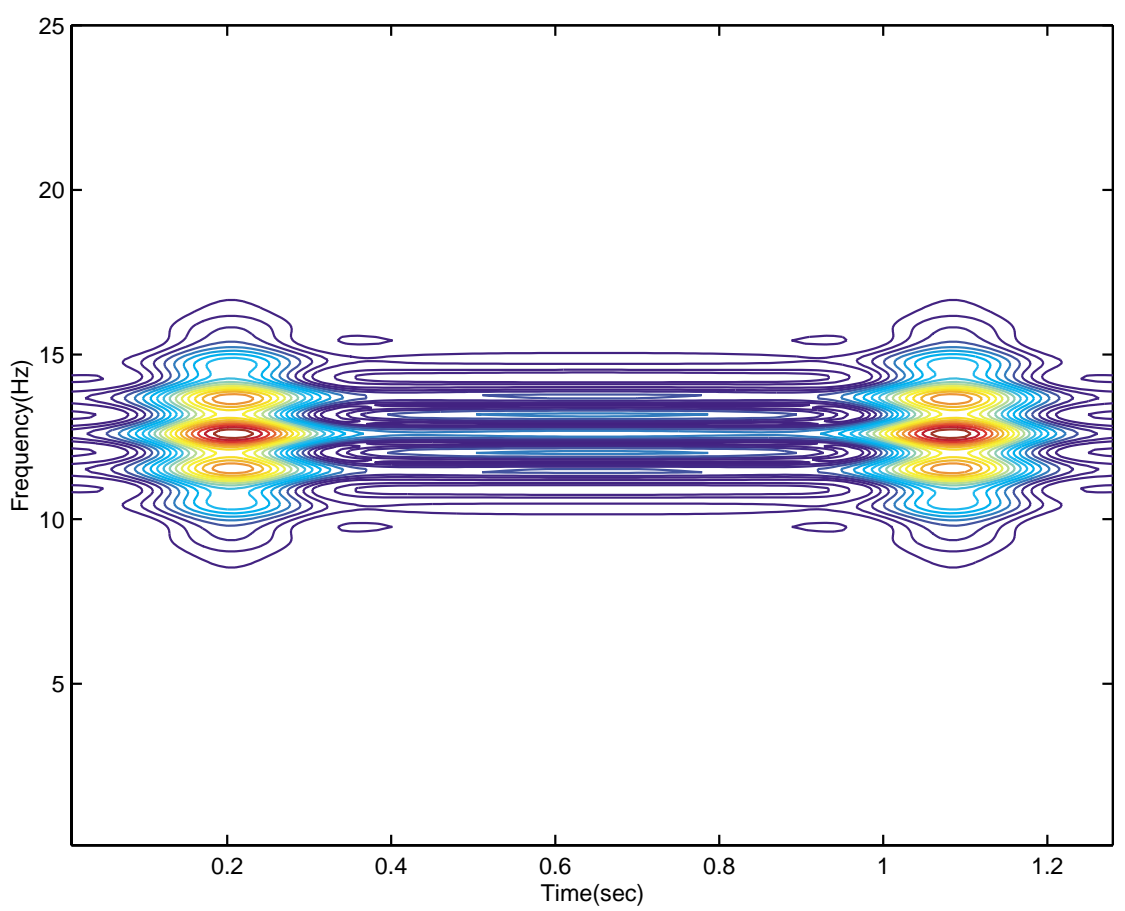

(b)

Fig. 3. Comparison between the WVD and SWFOMS for the two component signals centered at $0.2 \mathrm{~s}$ and $1.08 \mathrm{~s}$ with $12.5 \mathrm{~Hz}$. (a) The WVD weighted by an exponential kernel. (b) The SWFOMS weighted by an exponential kernel. 


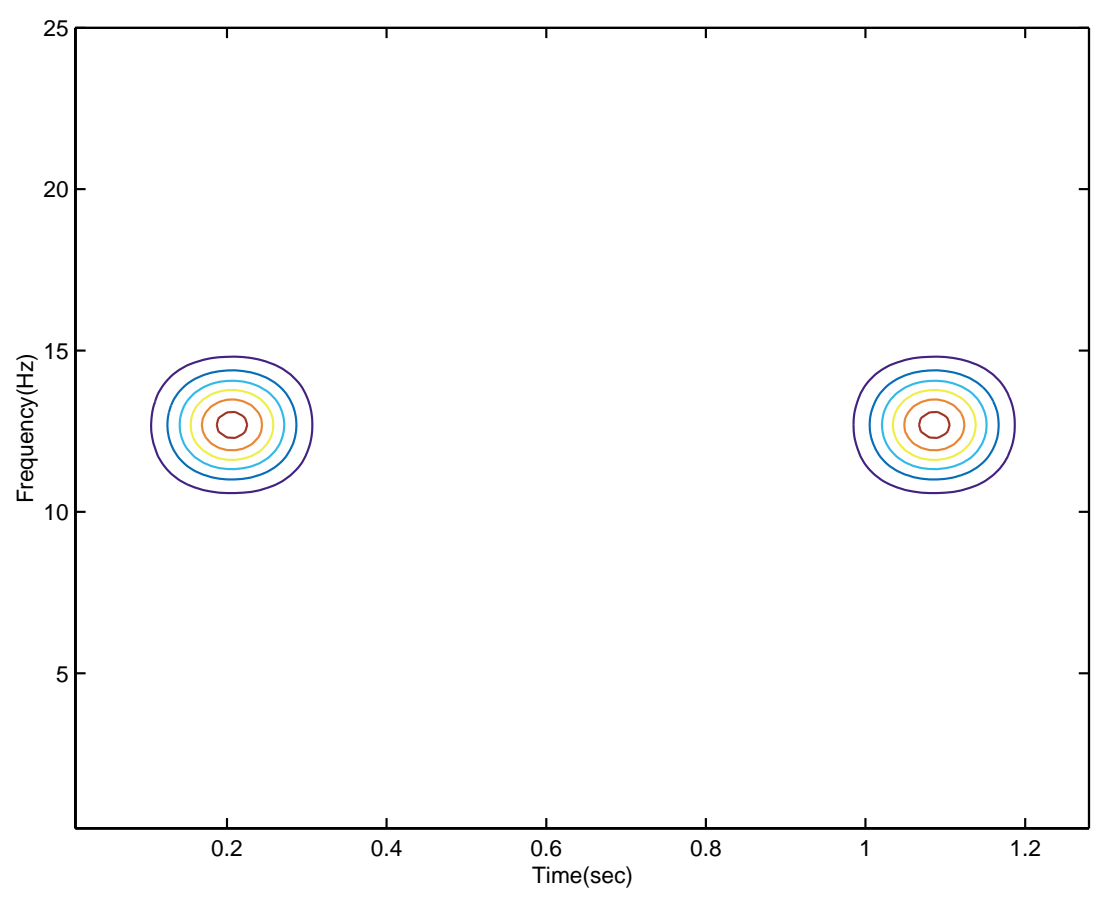

Fig. 4. The SWFOMS smoothed by the $\gamma$-method for the two component signals centered at $0.2 \mathrm{~s}$ and $1.08 \mathrm{~s}$ with $12.5 \mathrm{~Hz}$.

$$
\begin{aligned}
= & \int_{\xi} H\left(-\frac{\xi}{2}\right) H^{*}\left(\frac{\xi}{2}\right) S^{*}\left(f+\frac{1}{2} \xi\right) \\
& S\left(f-\frac{1}{2} \xi\right) e^{-j 2 \pi \xi t} d \xi \\
= & \frac{1}{2} \int_{\xi^{\prime}} H\left(-\xi^{\prime}\right) H^{*}\left(\xi^{\prime}\right) S^{*}\left(f+\xi^{\prime}\right) \\
& S\left(f-\xi^{\prime}\right) e^{-j 2 \pi \xi^{\prime}(2 t)} d \xi^{\prime}
\end{aligned}
$$

where $\xi^{\prime}=\xi / 2$. Using convolution, Eq. (9) may be written as follows:

$$
W_{p w}(t, f)=s_{h}^{*}(f, 2 t) \underset{t}{*} s_{h}(f, 2 t)
$$

where $s_{h}(f, t)$ is the short time Fourier transform (STFT), Therefore, the PWD can be written as,

$$
\begin{aligned}
W_{p w}(t, f) & =s_{h}^{*}(f, 2 t) * s_{t}(f, 2 t) \\
& =\int_{-\infty}^{\infty} s_{h}^{*}(f, t+\tau) s_{h}(f, t-\tau) d \tau
\end{aligned}
$$

In order to emphasize the auto-terms of the PWD for a multi-component signal, a window function $\gamma(\tau)$ can be incorporated into Eq. (11) and the smoothed Wigner distribution (SWD) can be developed as follows:

$$
\begin{aligned}
& W_{2, s w}(t, f) \\
= & \int_{-\infty}^{\infty} \gamma(\tau) s_{h}^{*}(f, t+\tau) s_{h}(f, t-\tau) d \tau
\end{aligned}
$$

We refer to this window function as the $\gamma$ method. In Eq. (12), when $\gamma(\tau)=1$, the SWD becomes the WVD and when $\gamma(\tau)=\delta(\tau)$ the SWD becomes the spectrogram. Therefore, in order to smooth the cross-terms of the WVD for the signal of the form of Eq. (3), the duration $T_{\gamma}$ for $\gamma(\tau)$ needs to be selected in accordance with,

$$
T_{h}<T_{\gamma}<\min _{i, j} \frac{t_{i}-t_{j}}{2}-T_{h}
$$

where $t_{i}$ and $t_{j}$ are the temporal positions of the signal components and $T_{h}$ is the duration of $h(t)$. A pictorial explanation for Eq. (13) can be seen with reference to Fig. 1. Consider the convolution of $s_{h}(f, 2 t)$ and $s_{h}^{*}(f, 2 t)$ using a rectangular window $\gamma(t)$. In order that only the auto-terms are convolved, the condition Eq. (13) should be satisfied as shown in Fig. 1(c); otherwise the cross-terms will also be included in convolution, as shown in Fig. 1(d). The SWD is obtained by convolving two signals $s_{h}(f, 2 t)$ and $s_{h}^{*}(f, 2 t)$ with respect to time and using the $\gamma$-method for smoothing the cross-terms in Eq. (12). Similarly, a smoothed version of the SWFOMS also can be obtained by the convolution of two SWD $W_{2, s w}(f, 2 t)$ and $W_{2, s w}^{*}(f, 2 t)$ with respect to time as follows:

$$
\begin{aligned}
& W_{4, s w f o m s}(t, f)= \\
& \frac{1}{2} \int_{\tau} \gamma(\tau) W_{2, s w}(f, t+\tau) \cdot W_{2, s w}^{*}(f, t-\tau) d \tau
\end{aligned}
$$




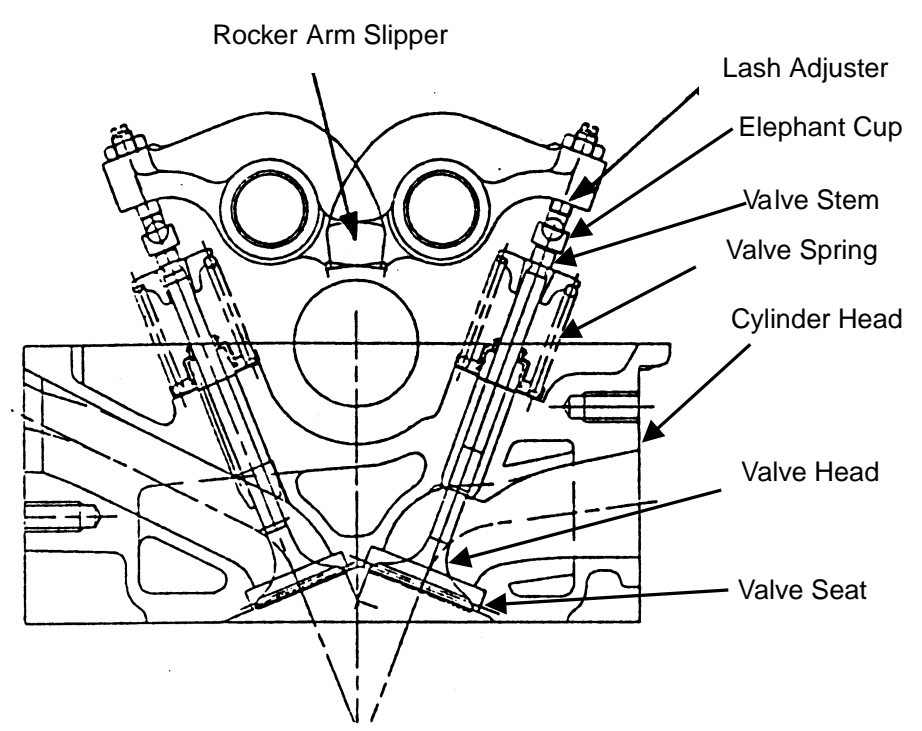

Fig. 5. Valve train system and cylinder head of the 2.0 litre, in-line 4 cylinder engine used in this test.
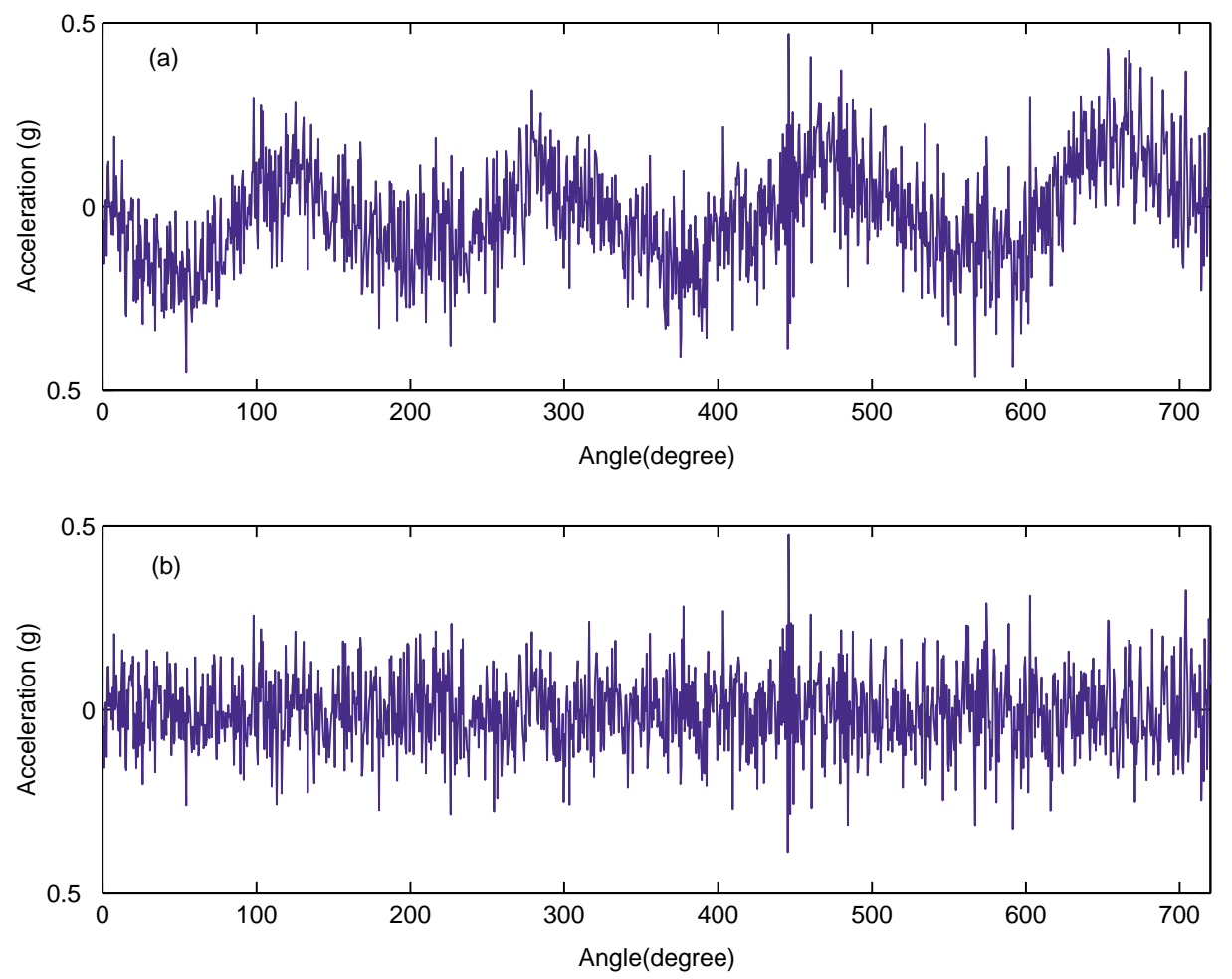

Fig. 6. Results of enhancement for measured data from an automotive engine with abnormal condition of valve system. (a) Time series data. (b) Enhanced data after two stage ALE.

\section{Numerical example}

This theoretical observation is verified via a simulation, the results of which are shown in Fig. 2. In
Fig. 2(a), The SWFOMS is computed for a 128 point time series at an assumed sampling rate of $100 \mathrm{~Hz}$. The signal contains two components occurring at different times, 0.2 and $1.08 \mathrm{~s}$, but at the same frequency, 


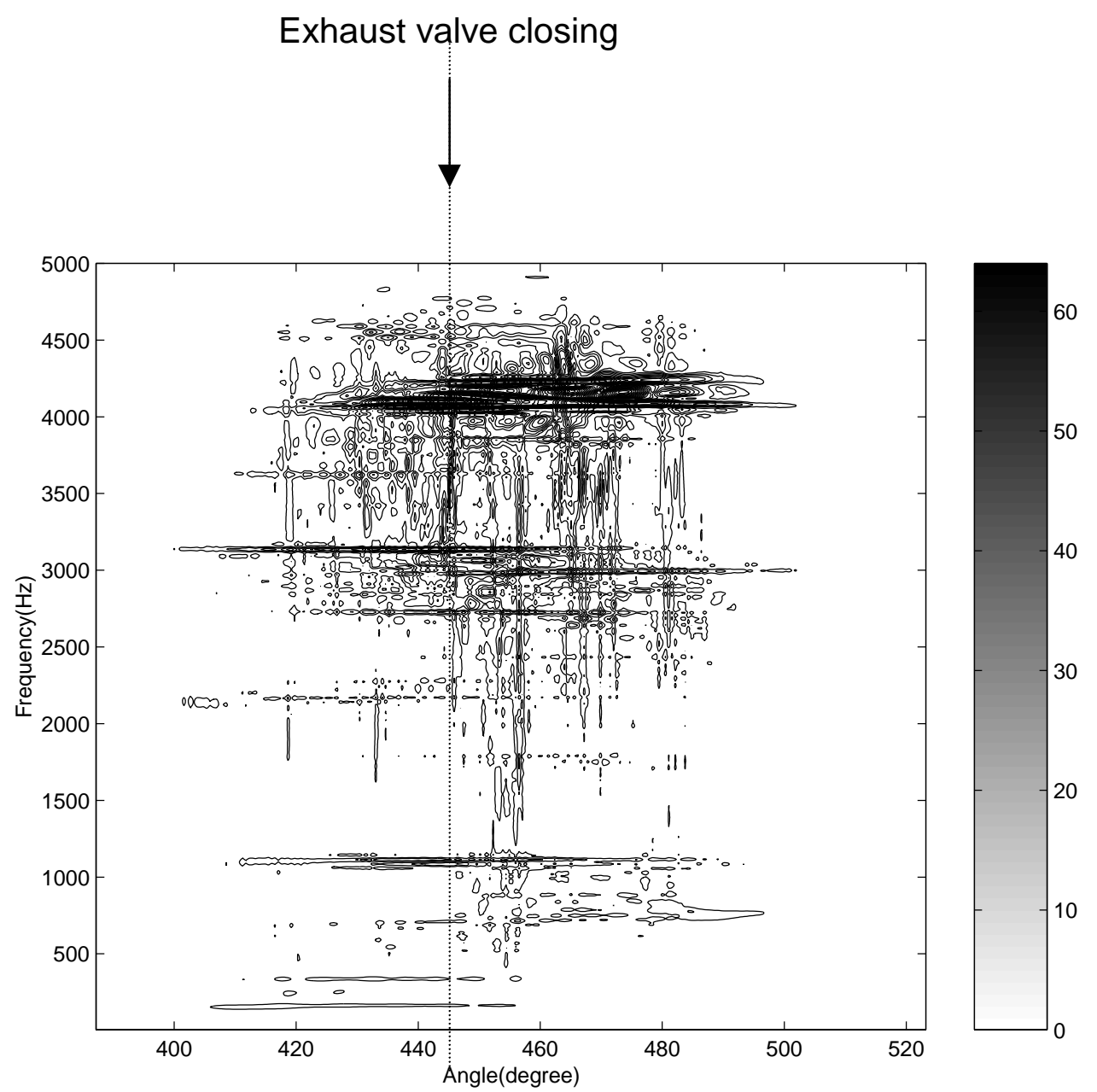

Fig. 7. Sliced Wigner forth order moment spectra smoothed by the exponential kernel for the enhanced signal after two stage ALE.

$12.5 \mathrm{~Hz}$. In this case there are three sets of cross-terms, in contrast to a WVD that would only generate a single set of cross-terms as shown in Fig. 2(b). The SWFOMS cross-terms appear at quarter intervals between the two components. The off-center cross-terms at 1/4th and $3 / 4$ ths of the interval are oscillatory albeit only in the frequency direction. However, the cross-terms appearing at mid point contain both an oscillatory element and a non-oscillating element. Figure 3 shows the results of applying the exponential kernel to the data depicted in Fig. 2. The results of this simulation are less satisfying since the exponential kernel is poorly suited to removing cross-terms since it is impossible to smooth nonoscillating cross-terms by the exponential kernel function as shown in Fig. 3(a). Even the WVD weighted by exponential kernel cannot remove the cross term perfectly as shown in Fig. 3(b). The effectiveness of smoothing the SWFOMS using the $\gamma$-method is again demonstrated via a simple simulation. Figure 4 depicts the SWFOMS calculated for the same data set as used to compute Fig. 2 using the $\gamma$-method. In this case, the sampling frequency is $100 \mathrm{~Hz}$, the number of samples is 512 , and $t_{i}-t_{j}=0.88 \mathrm{~s}$. The width of window for $\gamma(\tau)$ is $0.12 \mathrm{~s}$, and the duration of the sliding window $h(\tau)$ with a bandwidth of $50 \mathrm{~Hz}$ is $0.64 \mathrm{~s}$. According to these results, the non-oscillating cross-terms can be eliminated, as shown in Fig. 4.

\section{Application}

The engine used in this test was a 2.0 litre, in-line 4-cylinder engine. The engine was operating at a nominal idling speed of $800 \mathrm{rpm}$. One microphone was used to make measurements in the engine compartment. This microphone was calibrated with a B\&K 


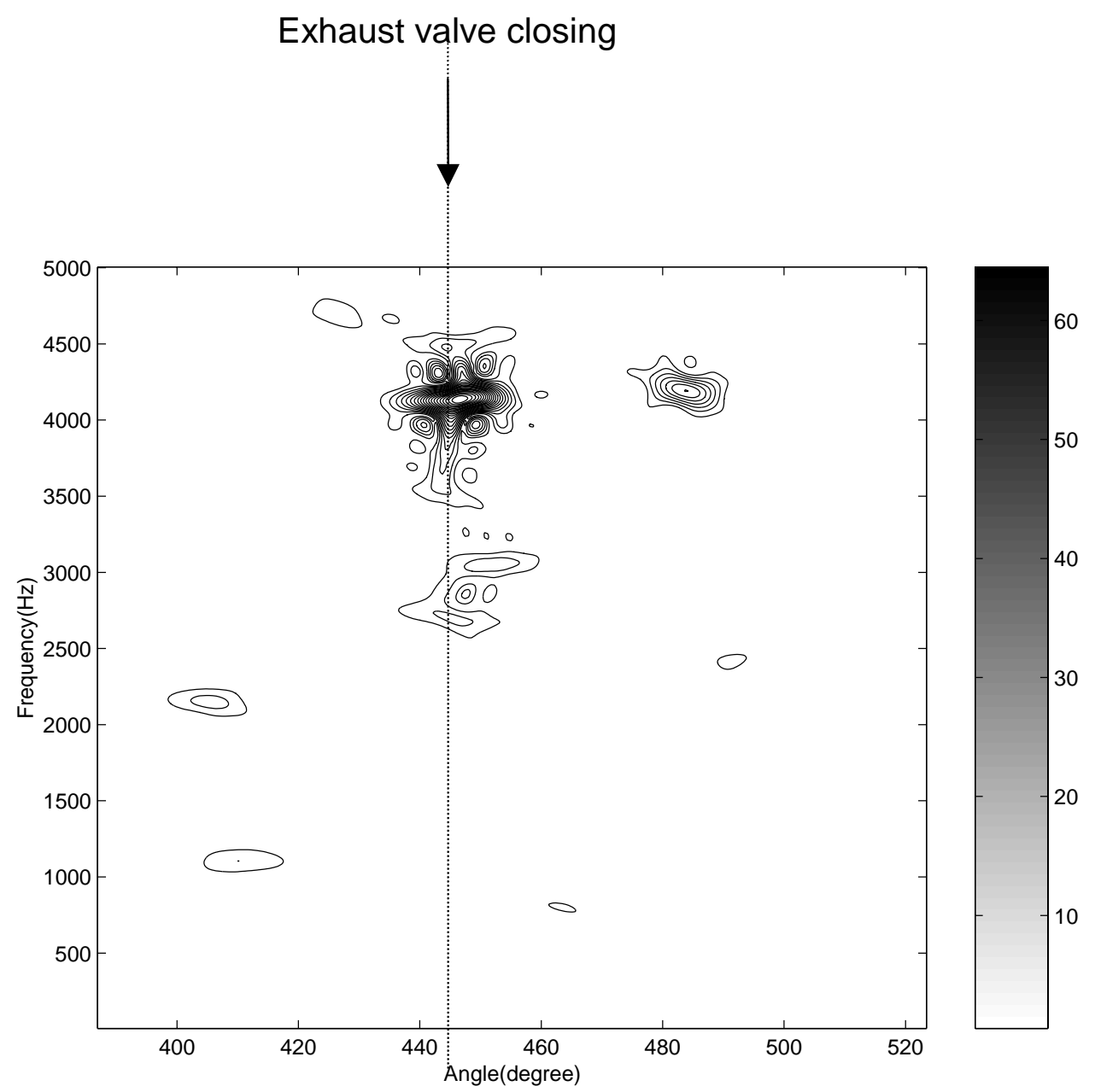

Fig. 8. Sliced Wigner forth order moment spectra smoothed by the $\gamma$-method for the enhanced signal after two stage ALE.

pistonphone $[94 \mathrm{~dB}(\mathrm{~A})]$. The measured analogue data were converted to digital form at a sampling rate of $10 \mathrm{kHz}$. Figure 5 shows the drawing of the valve train system and cylinder head of the test engine. A lash adjuster screw controls the contact force between the elephant cup of the lash adjuster and the valve stem of the valve and the equivalent stiffness of the valve system. In the engine production line, impulsive sounds are introduced by incorrectly setting the lash adjuster screw out of range of the design value $[9,19]$. This wrong setting affects not only the dynamic behaviour of the valve system but also the performance of the engine [6]. These impulsive sounds are generated as the valve head impacts on the valve seat of the cylinder head when the valve opens and closes. However, these impulsive sounds are immersed in the high level of background noise, such as integer multiples of fundamental rotation speed and broadband random noises [2]. Figure 6(a) shows the signal measured from the microphone. These results show that the impulsive sounds are immersed in the background noise. In order to enhance these impulsive sounds, the two-stage ALE (Adaptive Line Enhancer) has been employed [2]. Figure 6(b) shows the impulsive sounds enhanced by the two-stage ALE. After the two-stage ALE, the harmonic noises of engine rotation and pure tone noises are nearly cancelled. However, making an objective measurement of impulsive sounds still tends to be difficult because of the high level of broadband random noise. In addition, these impulsive sounds are non-stationary in nature and one cannot readily use the frequency domain representation to identify the temporal location of an event. For the time-frequency analysis of this non-stationary impulsive sound immersed in the broadband random noise, the SWFOMS, which is a more robust time-frequency method in the presence of random (Gaussian) noise, has 
been employed. Figure 7 shows the SWFOMS analysis smoothed by an exponential kernel function, which is one of conventional kernels, for these non-stationary impulsive sounds immersed in the broadband random noise. The light bar expresses the relative magnitude to maximum magnitude with 64 ranks of grey colour. However, it is difficult to identify the impulsive sounds because of non-oscillating cross-terms among the impulsive sounds. If the SWFOMS smoothed by the $\gamma$ method is applied, this Fig. can be clarified as shown in Fig. 8. Again, this demonstrates that the first dominant impulsive sound occurs at a crank angle of $445^{\circ}$. This impulsive sound, which has frequency components at $4200 \mathrm{~Hz}$ and $2800 \mathrm{~Hz}$, is due to the closing of an exhaust valve. The second dominant impulsive sound, which occurs at a crank angle of $485^{\circ}$, has frequency components at $4200 \mathrm{~Hz}$ and is one of the weak impulsive sounds due to other moving components of the test engine, such as piston motion, crankshaft, injector, etc.

\section{Conclusion}

A trial detecting the impulsive sounds occurring from faults of the valve system in an engine has been done. These impulsive sounds are immersed in the high level of background noise. The sliced Wigner fourth order moment spectra (SWFOMS) has been employed because of its robustness in the presence of random (Gaussian) noise. In the application of the SWFOMS, the non-oscillation cross-terms between impulsive sounds make it difficult to detect the impulsive sounds. The SWFOMS smoothed by $\gamma$-method has been developed in this paper to smooth these non-oscillation crossterms, and it has been successfully applied to the detection of valve system faults in an engine.

\section{Acknowledgments}

This work was supported by grant No. 2000-230400-004-3 from the Basic research Program of the Korea Science \& Engineering Foundation.

\section{References}

[1] A.C.M. Claasen and W.F.G. Mecklenbrauker, The Wigner distribution - A tool for time-frequency signal analysis: part I, II, III, Phil. J. Res. 35 (1980), 217-389.

[2] B. Fielding, Identification of mechanical sources of noise in a diesel engine, Ph.D. Thesis, Manchester University, 1968.

[3] D. Dyer and R.M. Stewart, Detection of rolling element bearing damage by statistical vibration analysis, Trans. of ASME. Journal of Mechanical Design 100 (1978), 229-235.

[4] G.D. Meegan, H.R. Nelson, M.L. Barlett and G.R. Wilson, Fault detection in engine through higher order spectra alalysis of acoustic signatures, Journal of the Acoustics Society of America 106 (1999), L1-L6.

[5] H.A. Gaberson, Application of Choi-Williams reduced Interference time frequency distribution to machinery diagnostics, Shock and Vibration 2 (1995), 437-444.

[6] H. Heisler, Valve Operating Condition, Advanced Engine Technology, Edward Arnold, 1995, pp. 11-40.

[7] J.R. Fonollosa and C.L Nikias, Wigner-Higher-Order Moment Spectra Definition, Properties, Computation and Application to Transient Signal Analysis, IEEE Transaction on Signal Processing 41 (1993), 245-266.

[8] L. Cohen, General Approach and the Kernel Method, Timefrequency analysis, Prentice Hall, 1995, pp. 136-197.

[9] O. Maeda, Quantitative evaluation of abnormal engine noise, Internal Combustion Engines 27 (1988), 343-340.

[10] Q. Zhuge, Non-stationary modelling of vibration signals for monitoring the condition of machinery, Mechanical Systems and Signal Processing 4 (1990), 355-365.

[11] R.H. Lyon, Impact as a source of vibration, Machinery Noise and Diagnostics, Butterworths, USA, 1987, pp. 24-43.

[12] R. Randall, A new method of modeling gear faults, Trans of ASME. Journal of Mechanical Design 104 (1982), 259-267.

[13] R. Randall, Cepstrum analysis and gearbox fault detection, $B \& K$ Technical Report (1981), 13-150.

[14] S.K. Lee and P.R. White, Fault diagnosis of rotating machinery using Wigner Higher Order Moment Spectra, Mechanical Systems and Signal Processing 11 (1997), 637-650.

[15] S.K. Lee and P.R. White, Impulsive sound analysis of automotive engine, Proceedings of 1997 Noise \& Vibration Conference (1997), SAE 970262.

[16] S.K. Lee and P.R. White, The enhancement of impulsive signals for fault detection, Journal of Sound and Vibration $\mathbf{2 1 3}$ (1998), 485-505.

[17] S.K. LEE, Adaptive Signal Processing and Higher Order Time Frequency Analysis and Their Application to Condition Monitoring, Ph.D. thesis, ISVR, The University of Southampton, UK, 1998.

[18] S.K. Lee, C.B Choi and S.D. Yeo, Identification of relation between crankshaft bending and interior noise of A/T Vehicle in idle state, Society of Automotive Engineering (1993), SAE 930618.

[19] T. Nemura, N. Adachi and K. Susuki, Research in regard to sensory characteristics measuring for the impulse noise of the engine valve system, Society of Automotive Engineering (1991), SAE 910620.

[20] W.J. Wang, Wavelet transform in vibration analysis for mechanical fault diagnosis, Shock and Vibration 3 (1996), 17-26. 

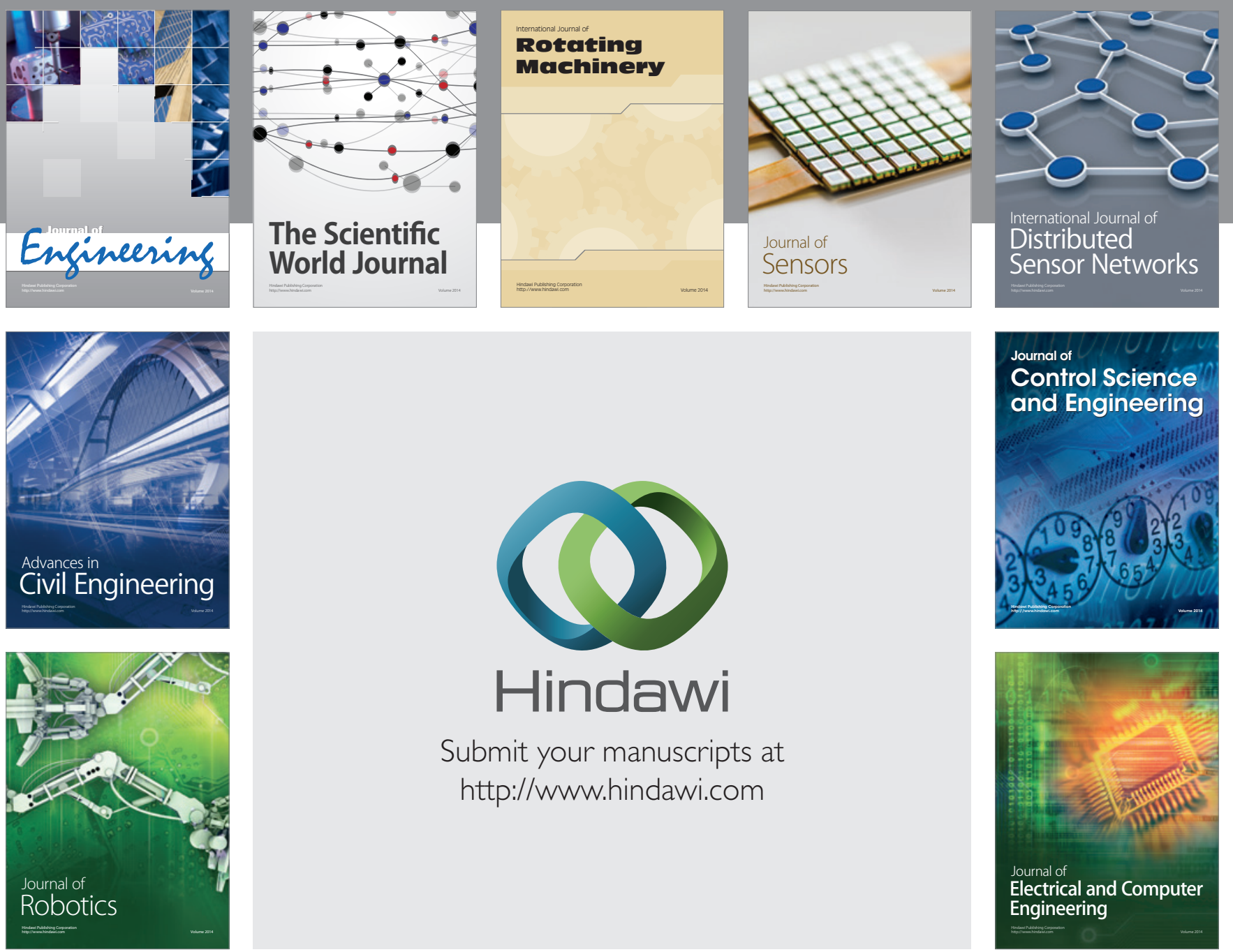

Submit your manuscripts at

http://www.hindawi.com
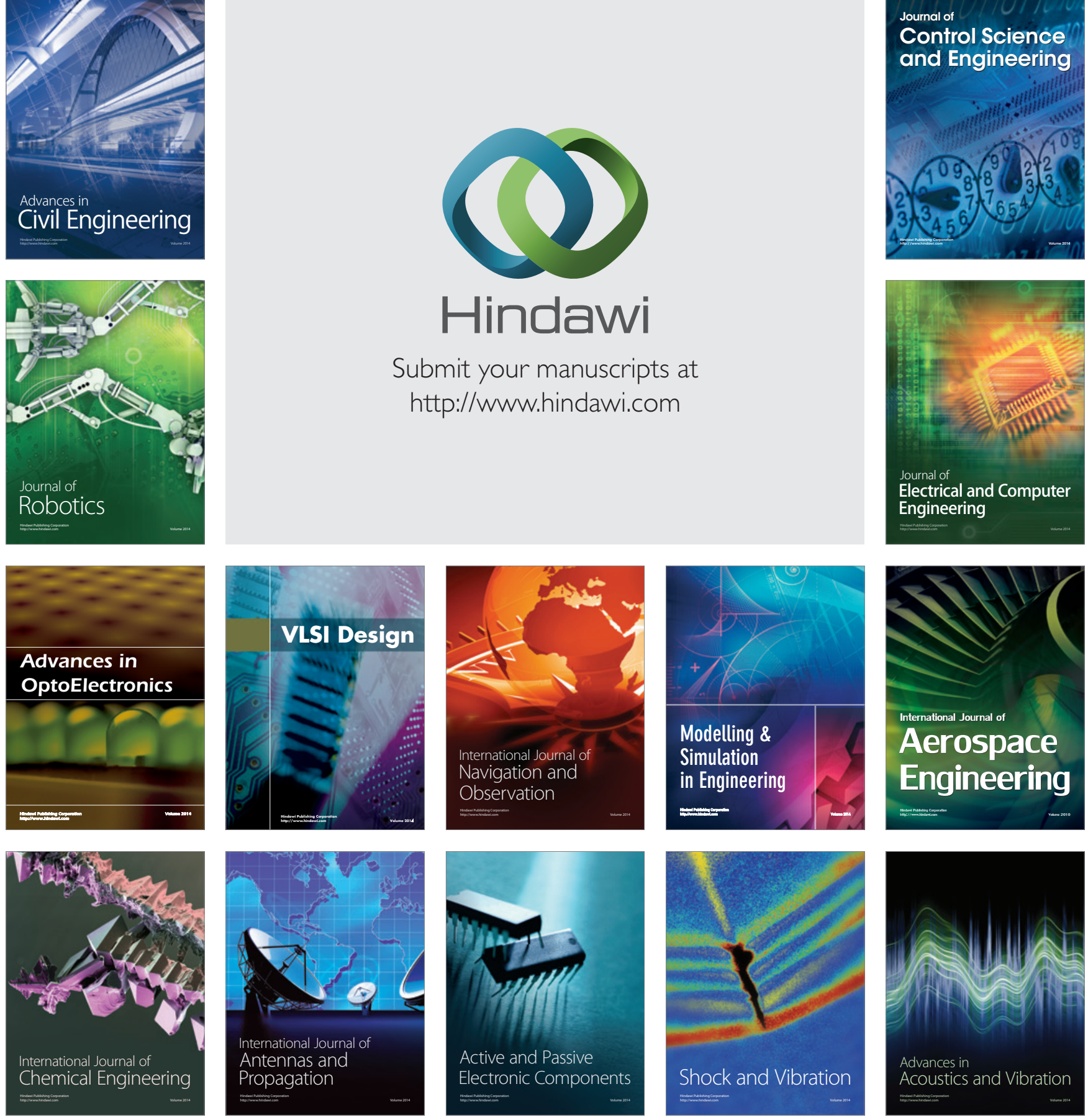